\title{
Microprocessor Power Analysis by Labeled Simulation
}

\begin{abstract}
In many applications, it is important to know how power is consumed while software is being executed on the target processor. Instruction level power microanalysis, which is a cycleaccurate simulation technique based on instruction label generation and propagation, is aimed at answering this question for a superscalar and pipelined processor. This technique requires the micro-architectural details of the CPU and provides the power consumption of every module (or gate) for each active instruction in each cycle. To validate the approach, a Zilog DSP core was designed by using a $0.25 \mu$ TSMC cell library and the power consumption per instruction was collected using a Verilog simulator specially written for the DSP core.
\end{abstract}

\section{INTRODUCTION}

Given the micro-architectural description of a target processor and some application program to be executed, it is usually useful to know which modules (or gates) consume the most power and under what input data or internal state conditions. For example, a common question is how to automatically identify and eliminate unwanted power consumption during the program execution by hardware control (e.g., clock gating) and/or software optimization (e.g., compilation). To fully answer the question, we need to know the cycle-accurate power consumption of each individual module (or gate) in the processor due to the execution of each instruction. We refer to this kind of analysis as power microanalysis, and present a simulation-based strategy to achieve it. Microprocessor designers to improve the power efficiency of a proposed instruction set architecture can use the power microanalysis report. Similarly, compilers can use power microanalysis to reduce the energy cost of an application program running on the target microprocessor by performing high-level transformations or lowlevel code generation.

Power microanalysis reports can also be useful in generating an accurate power macromodel of a processor [1][2]. A power macromodel is usually trained by running a number of instruction traces and studying the resulting power dissipation profile in the target circuit. Without an accurate breakdown of power consumption of each instruction in the pipeline, the various power dissipation effects have to be averaged out. These power effects include, for example, the power consumption caused by pipeline stalls, pipeline flushes, and cache misses. Furthermore, in some cases, power may be dissipated due to unwanted operations (this is mainly because of poor design practices). For example, the input operands of the multiplier may change even when the executed instruction is not a multiplication instruction, which in turn causes extra power consumption. If this kind of effect is not accurately modeled during the power macromodel construction, it will be treated as a random statistical variation at best, which will then increase the error of the power macromodel. The power microanalysis technique proposed here can be quite valuable in constructing an accurate instruction-level power macromodel because it provides information about the power consumption caused by each instruction in each gate in the circuit, while accounting for pipeline stalls, pipeline flushes, and cache misses.

The instruction execution in a modern CPU has the following characteristics:

- Multiple instructions are executed concurrently in the processor (e.g., VLIW and superscalar).

- Interactions between the instruction and the architecture can cause significant power consumption (e.g., Branch Misprediction).

- Interactions among the instructions greatly contribute to the overall power consumption of the CPU (e.g., Data dependency and Resource contention).

Because of this complexity, it is very difficult to automatically generate the equation form of the instruction-level power macromodel or even perform the calibration process (i.e., calculate the macromodel equation coefficients) for a given power macromodel equation form. For example, in [2], the macromodel equation is manually designed and then automatically calibrated by measuring the power dissipation of a set of specially designed instruction traces. ${ }^{~}$ Running an application program that is simply a loop with only one or at most two types of instructions typically generates the trace. The measured instruction power is called base cost, which is used for instruction-level macromodel training. The inter-instruction temporal effects can also be calculated and included in the model equation using these training traces. However, the model is still too simple to capture the actual CPU power dissipation. More precisely, because of the lack of detailed (module-level or gate-level) knowledge about the power consumption of each individual instruction in each clock cycle, the following difficulties arise:

- The initial power macromodel equation form (i.e., the number and meaning of different terms and the way they are combined) has to be input by the designer based on his experience and knowledge about the microprocessor architecture. If the initial form is incomplete or inappropriate, the accuracy of the power macromodel predictions will be adversely affected.

- It is very difficult to ensure proportionate coverage of the various power consumption factors in the processor (e.g., instruction mix and order, pipeline effects, and branch handling policy) by the macromodel equation. This calibration step requires detailed simulation of a very large number of complex instruction traces (i.e., with a number of instruction types and exercising different hardware conditions in the pipeline) to ensure correct calibration of the macromodel coefficients to cover instruction correlations, data dependencies, various architectural effects and scenarios. In contrast, with the aid of microanalysis power report, the macromodel calibration process will be a lot simpler since the required information is available.

Notice that our work is not intended to replace the work of [2] or other similar work, but to facilitate them by providing a detailed power report. Our technique handles both super-scalar and pipelined processors.

An instruction is active if it is being executed in the instruction pipeline of a given microprocessor. The power microanalysis for the microprocessor can be defined as identifying what active instructions cause the power consumption for each gate in an RTlevel description of the processor. A naïve approach simply assumes that the power consumption of every gate is caused by all of the active instructions. In this paper, we present a more sophisticated and significantly more accurate simulation-based technique called "Labelled Simulation" for evaluating the power consumption of the microprocessor. Note that although a detailed RT-level description of the microarchitecture is assumed in this paper, power microanalysis can be performed even when some parts of the processor are behaviorally specified as long as the complete model can be simulated.

This paper is organized as follows. Section II gives a detailed microanalysis model. Section III gives a DSP RTL design as an example. Section IV describes how to construct the experiment. Section V presents the result. Conclusions are given in Section VI.

\section{MICROANALYSIS MODELING A. Problem Formulation}

Assume that there are $n$ gates, $g_{1} \ldots g_{n}$, in the circuit description of the target processor, and $k$ instructions, $I_{1} \ldots I_{k}$, are active in the

1 Please refer to [3][4] for detailed reviews of high-level (including software-level) power estimation and optimization. 
processor in a certain clock cycle. We find a labeling $L_{i}=\left\{I^{\prime}{ }_{1}, I_{2}^{\prime}\right.$, $\left.I_{3}{ }_{3} \ldots\right\}$ for each gate $g_{i}, i=1 \ldots n$, such that the energy consumption of $g_{i}$ in the current clock cycle is caused by instructions in $L_{i}$. If $L_{i}$ is empty, the energy consumption of $g_{i}$ is not caused by any particular instruction and is considered as the intrinsic energy consumption of the processor (e.g., the energy consumption of the instruction cache is not caused by an individual instruction). If $L_{i}$ contains multiple instructions, the energy consumption of $g_{i}$ is equally caused by all of the instruction in $L_{i}$.

Define $G(I)$ as a set of gate indices such that instruction $I$ belongs to the label of each gate according to the indices in $G(I)$.

$$
G(I) \equiv\left\{i \mid I \in L_{i}\right\}
$$

The energy consumed by instruction $I$ in the current clock cycle is:

$$
E(I)=\frac{1}{2} \sum_{j \in G(I)} \frac{1}{\left|L_{j}\right|} C_{j} V_{d d}^{2} s w_{j}
$$

where $s w_{j}=1$ if wire $j$ switches, otherwise $s w_{j}=0$; and $C_{j}$ is the effective capacitance of gate $j$. The total energy dissipation of an instruction $I$ for the program being evaluated is calculated by the summation of $E(I)$ over clock cycles when $I$ is active (non-empty $G(I))$. Note that the labels need to be updated every clock cycle while the instruction is propagating through the pipeline.

Consider a simple MIPS-like instruction pipeline with five stages, and assume that there is no feedback path between any two pipelines. In this case the labeling problem is solved by propagating the labels from one pipeline stage to next through the labeling network, which is equivalent to RT or gate level logic network of the processor. The on-chip memory is treated in the same way as the flip-flops because its functionality is the same as that of the flip-flops (registers). The labeling can be derived by labeling the wires connected to the instruction memory (IM) as newly fetched instruction $I_{i}$ and propagating the labels in the network according to these rules:

Combinational gate: If we assume that the instruction pipeline has no feedback, the input labels of a gate will not contain different instructions. We simply pass the input label to the output.

Flip-flop: At the positive or negative clock edge, we label the flipflop itself with its input instruction label.

\section{B. $\quad$ Labeling Network}

To initialize the labeling propagation, the first task is to identify the label sources and sinks for label propagation.

- Source and Sink

Definition: The source refers to the set of gates (or wires) from which the labels are originated.

Definition: The sink refers to the set of gates (or flip-flops) where the instruction label is dropped.

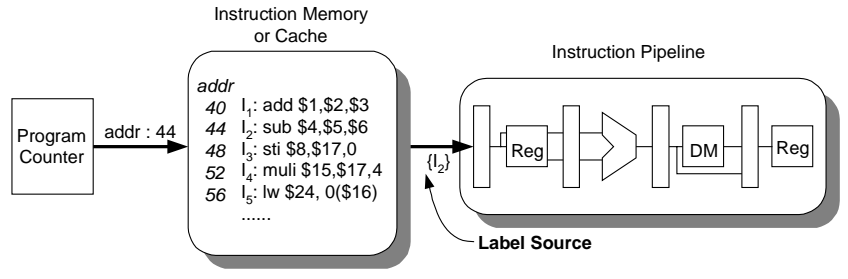

Figure 1 Instruction memory as the label source.

When a processor fetches an instruction $I$ from the external memory, cache, or on-chip memory, the set of wires connected to the read port are labeled as $L=\{I\}$. In , for example, the instruction addressed by the program counter, whose content is 44 , is fetched and the instruction bus is labeled as $\left\{I_{2}\right\}$. Sometimes, the instruction fetch unit is designed to fetch $k$ instructions in one clock cycle (e.g. VLIW machine), then the read port of instruction memory (or cache) is labeled by those instructions, $\left\{I_{1}, \ldots, I_{k}\right\}$. Note that for some advanced processors, there may be multiple instruction memories in the system. Therefore, the label source may not be unique. The new instruction labels continuously flow into the system from the label sources in every clock cycle.

The next question is when we should stop propagating an instruction label or drop an instruction from a label in the network. The instruction label is only removed when an instruction label, which is stored in a flip-flop, is not transferred to any other flipflop in the processor (including the flip-flop where it is stored). For example, if an instruction label is propagated to the last stage of the pipeline and if this label is not transferred to data paths in the processor, it will be overwritten by another label in the next clock cycle. When an instruction label is transferred into the onchip memory or register file, the question arises whether we should label the memory elements inside the memory or the register file. Note that if the labels are not removed in these memory elements (flip-flops or memory cells), the number of distinct instructions in all of the labels in a given clock cycle may be larger than the number of pipeline stages. As an example, in Figure 2, a 'mov' instruction (denoted as $I_{m o v}$ ) finishes its job after writing immediate value 100 to a register. Then instead of propagating $\left\{I_{m o v}\right\}$ after we write to the register file, the label should be dropped because the 'mov' instruction never uses the written data again. After a number of clock cycles, the register content may be used by another instruction 'add $\$ 3, \$ 1, \$ n$ '. However, the energy consumption induced by the newly fetched register content $(\$ 1)$ should be attributed to the instruction that fetched the register (i.e., add $\$ 3, \$ 1, \$ n$ ), not to the instruction that wrote it (i.e., mov \$1,100). Similarly, when a 'store' instruction writes some data into the data memory, it never uses the memory content again and the label should be dropped in the memory.

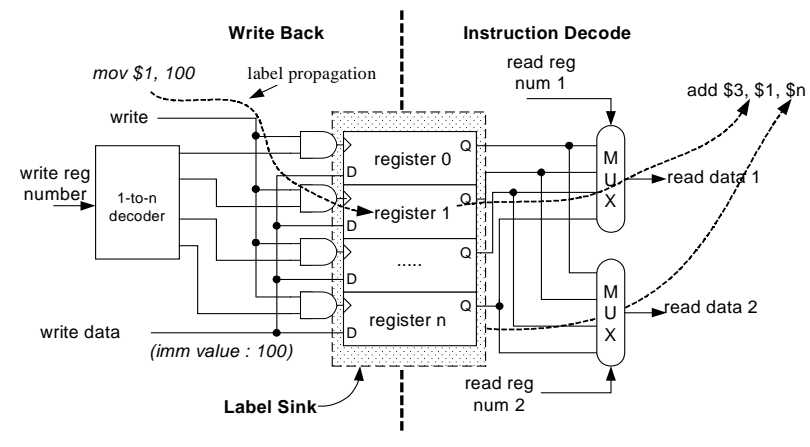

Figure 2 A 2-read, 1-write register file.

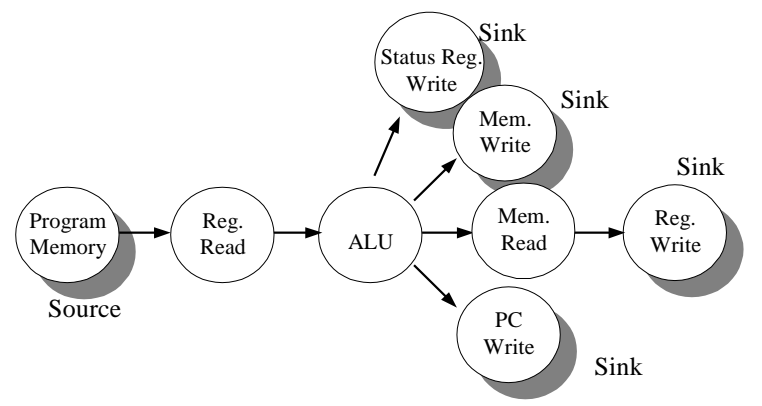

Figure 3 Instruction label flow chart for MIPS.

In MIPS architecture, the register file, data memory, status register, and program counter are marked as the label sinks. Note that the contents of the flip-flops or memory elements that are marked as label sinks may affect the power consumption of other modules in the system. In general, labels are dropped one clock 
cycle after when they reach a sink. This is required, for example, when the outputs of the $\mathrm{X}, \mathrm{Y}, \mathrm{P}$, and Accumulator registers in the DSP chip shown in Figure 12 connect to other modules (i.e., multiplier, shifter, or buses) directly and cause extra power consumption even though the labeled instruction has finished its task. It is also possible that instructions require different definitions for label sink locations that may conflict with each other. If such a conflict occurs, instruction $I$ is dropped from the label if it reaches a node where the node is defined as a sink for $I$.

Figure 3 shows the journey of an instruction in the pipeline of MIPS architecture. The lifetime of an instruction starts from the source and ends at the sink (if it is not discarded in the middle, e.g., due to a pipeline flush). At each clock cycle, the instruction label moves toward the label sink and activates some control signals or simply stays in the same place in the case of encountering a control or a data hazard.

\section{- Propagation Rule}

After synthesizing and mapping a RTL design to a standard cell net-list, the instruction label starts from instruction memory and propagates through nets and cells under a specific propagation rule. Each distinct type of standard cell should have an associated propagation rule. For a simple inverter, we propagate its input label to its output. For a 2-input gate, the notation is shown in Figure 4, where $\mathrm{in}_{1}$ and $\mathrm{in}_{2}$ denote the logic values of the inputs. The rule is that if only one of $L_{1}$ or $L_{2}$ is empty, than the nonempty label will be propagated. That means $L_{\text {out }}=L_{1}$ if $L_{2}=\{\}$ and $L_{\text {out }}=L 2$ if $L_{l}=\{\}$.

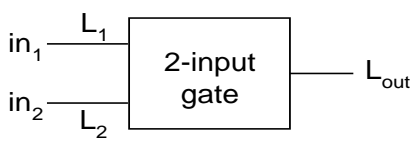

Figure 4 Labeling for 2-input gates.

The following table lists the label propagation rules for the case that both $L_{l}$ and $L_{2}$ are non-empty. The rule is then as follows: $L_{l}$ will be propagated if $i n_{2}$ has a non-controlling value, and $L_{2}$ will be propagated if $i n_{l}$ has a non-controlling value. For OR gate:

\begin{tabular}{|c|c|c|}
\hline in1 & in2 & $L_{\text {out }}$ \\
\hline 0 & 0 & $L_{l}+L_{2}$ \\
\hline 1 & 0 & $L_{1}$ \\
\hline 0 & 1 & $L_{2}$ \\
\hline 1 & 1 & $L_{l}+L_{2}$ \\
\hline
\end{tabular}

The $L_{\text {out }}$ can be statically decided if $L_{1}=\{\}, L_{2}=\{\}$ or $L_{l}=L_{2}$. A similar propagation rule tables for primitive gates like AND gate, XOR gate, or NOR gate etc. could be easily built. However, for a combinational circuit cell like multiplexer, the propagation rule table derived from its equivalent Boolean implementation in terms of AND and OR gates should be consistent with the one from explicit derivation. We derive the propagation rule table from the explicit method. The logic values of multiplexed inputs are not important in this case, compared with OR gate. Instead, the select signal would play a major role. Consider a 1-bit 2-to-1 MUX with select logical value zero for label zero $\left(L_{0}\right)$ input and one for label one $\left(L_{1}\right)$ input. The following table shows the propagation rules.

\begin{tabular}{|c|c|c|c|c|}
\hline select & $L_{s}==\phi$ & $L_{0}==\phi$ & $L_{l}==\phi$ & $L_{\text {out }}$ \\
\hline 0 & $\mathrm{X}$ & $\mathrm{T}$ & $\mathrm{X}$ & $L_{s}$ \\
\hline 0 & $\mathrm{~T}$ & $\mathrm{~F}$ & $\mathrm{X}$ & $L_{0}$ \\
\hline 0 & $\mathrm{~F}$ & $\mathrm{~F}$ & $\mathrm{X}$ & $\left(L_{s}+L_{0}\right)$ \\
\hline 1 & $\mathrm{X}$ & $\mathrm{X}$ & $\mathrm{T}$ & $L_{s}$ \\
\hline 1 & $\mathrm{~T}$ & $\mathrm{X}$ & $\mathrm{F}$ & $L_{l}$ \\
\hline 1 & $\mathrm{~F}$ & $\mathrm{X}$ & $\mathrm{F}$ & $\left(L_{s}+L_{l}\right)$ \\
\hline
\end{tabular}

where ' $L==\phi$ ' is ' $\mathrm{T}$ ' if $L$ is empty, ' $\mathrm{F}$ ' if not empty. ' $\mathrm{X}$ ' denotes don't care condition. By observation, $L_{\text {out }}$ can be statically decided if exactly one of the $\left\{L_{0}, L_{l}, L_{s}\right\}$ is not empty or $L_{0}$ equals $L_{l}$.

For '+' operation between labels, we define two types of rules.

Definition: Priority Rule (Time-Stamp Rule)

If $L_{l}=\left\{I_{i}\right\}, L_{2}=\left\{I_{j}\right\}$, then $L_{1}+L_{2}=\left\{I_{\max (i, j)}\right\}$. Only the instruction that is fetched later (i.e. it has a larger time stamp) is kept in the merged label. Therefore, the labels after merge contain at most one instruction. In this rule, the instruction that is fetched later always assumes the responsibility for the power consumption when multiple instructions are propagated to the same wire.

\section{Definition: Union Rule}

$L_{1}+L_{2}=L_{l} \cup L_{2}$. In this rule, instructions that run into each other assume equal responsibility for the power consumption.

As mentioned in problem formulation, the input labels of a gate will not contain different instructions because of the assumption that there is no pipeline feedback. However, for a modern microprocessor, resource hazard is resolved automatically with a hazard detection unit, the pipeline-stall and flush mechanism or a data-forwarding unit etc. Those abilities require feedback information between different pipeline stages. Hence, the input labels could be annotated with different instructions. Several architectural patterns must be defined and analyzed for a specific microprocessor in order to make sure the propagation rules of the cells satisfy all the architectural patterns.

\section{C. $\quad$ Architecture Patterns}

We define an architecture pattern to have three fields as follows:

1. Name is a handle that we can use to describe the intended architecture effect (e.g., control hazard).

2. Description explains how the pattern is caused and how the processor reacts to the pattern.

3. Required Rule specifies how the propagation rule should work in response to the pattern.

The most common architecture patterns, pipeline-stall, data forwarding, and pipeline-flush, will be given as examples. Each pattern is caused by a certain architectural effect, and the related control circuitry will be explained. The required rule is given based on the specific control circuitry. The example is, however, representative, and other causes of an architecture pattern will give rise similar rules. Furthermore, the circuit implementation may vary for different processors, but the underlying structure for the instruction dispatch and routing will be similar.

\section{- Pipeline-stall Pattern}

Name: Pipeline-stall

Description: A data hazard usually occurs when an operation needs operands that are not computed or have been computed but are not yet available to the instruction. This is also called the "read-after-write" hazard. There are many other types of data hazards, depending on the target architecture. In particular, the super-scalar processors that perform speculative execution have complex control logic or architecture to make sure that the program works the same as when it is run on a scalar machine. Such complex architectures usually generate a lot of data hazards.

Figure 5 shows hoe the pipeline stall architecture injects bubbles into the instruction pipeline. If no hazard is detected, the MUX1/MUX2 select line is ' 0 ' and the instruction pipeline works as a streamlined pipe. If a hazard is detected, the MUX1/MUX2 select line equals ' 1 ' and $I_{4}$ is retained in flip-flops FF1, and a bubble is injected to flip-flops FF2. The hazard detection logic can be implemented as in Figure 6 , where '==' gate compares the inputs and produces ' 1 ' if the two inputs are equal. Figure 6 shows only part of the circuit; a complete hazard detection unit should 
compare both source operands of $I_{4}$ with the destination operands in the pipeline.

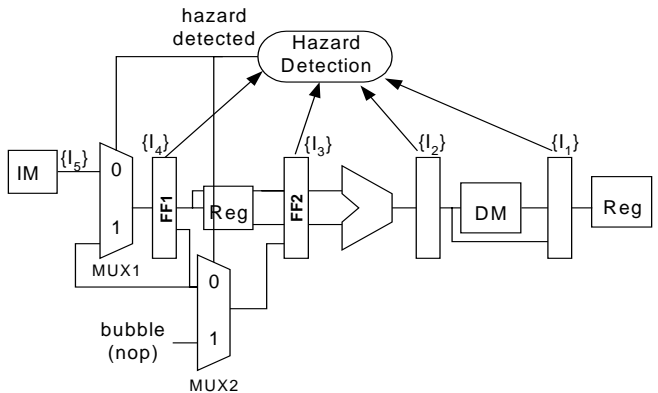

Figure 5 Pipeline-stall architecture.

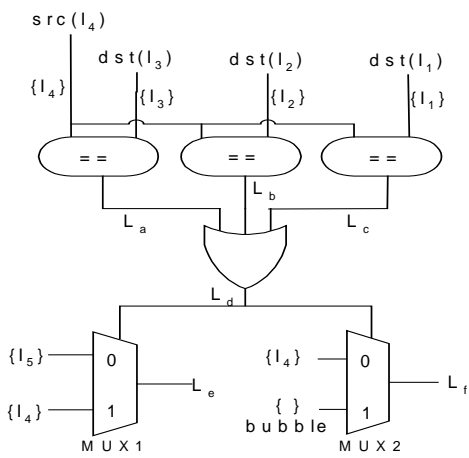

Figure 6 Hazard detection logic.

Required Rule (c.f. Figure 6):

$L=$ ' 1 ' denotes the labeled wire with logic value ' 1 '.

- $\quad L_{a}=\left\{I_{4}, I_{3}\right\} . \mathrm{L}_{\mathrm{b}}$ and $L_{c}$ follow similar rules.

- $L_{d}$ should be the minimal set while satisfying the following rules:

$$
\begin{array}{ll}
\circ & L_{d} \supseteq L_{a} \text { if } L_{a}=\text { ' } 1 \text { '. } \\
\circ & L_{d} \supseteq L_{b} \text { if } L_{b}=\text { ' } 1 \text { '. } \\
\circ & L_{d} \supseteq L_{c} \text { if } L_{c}=\text { ' } 1 \text { '. }
\end{array}
$$

- $L_{e}=\left\{I_{4}\right\}+L_{d}$ and $L_{f}=L_{d}$ if $L_{d}={ }^{\prime} 1$ '. Otherwise $L_{e}=\left\{I_{5}\right\}+L_{d}$ and $L_{f}=\left\{I_{4}\right\}+L_{d}$.

\section{- Data-forwarding Pattern}

\section{Name: Data-forwarding}

Description: Instead of stalling the pipeline to avoid data hazards, a data-forwarding architecture can be used to reduce the "readafter-write" hazard. In Figure 7, such architecture for the MIPS pipeline is shown. When there is read-after-write dependency between $I_{3}$ and $I_{2}$ or $I_{3}$ and $I_{1}$, then the operands required by $I_{3}$ can be directly forwarded from the computed result of $I_{2}$ or $I_{1}$. A forwarding unit can be implemented as shown in Figure 8.

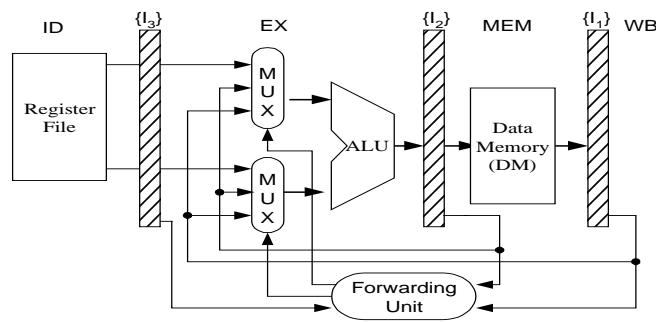

Figure 7 Data-forwarding architecture.

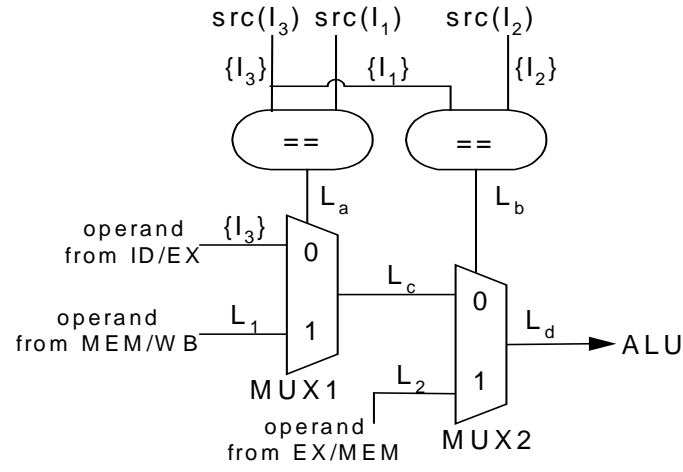

Figure 8 Data-forwarding control circuitry.

Required Rule: (c.f. Figure 8):

- $L_{a}=\left\{I_{3}, I_{1}\right\}$

- $L_{b}=\left\{I_{3}, I_{2}\right\}$

- $L_{c}=\left\{I_{1}\right\}+\left\{I_{3}\right\}+L_{1}$, if $L_{c}=$ ' 1 ', otherwise $L_{c}=\left\{I_{3}\right\}+\left\{I_{1}\right\}$

- $L_{d}=\left\{I_{3}\right\}+\left\{I_{2}\right\}+\left\{I_{1}\right\}$, if $L_{a}=$ ' 0 ' and $L_{b}=$ ' 0 '

$L_{d}=\left\{I_{3}\right\}+\left\{I_{2}\right\}+\left\{I_{1}\right\}+L_{l}$, if $L_{a}=$ ' 1 ' and $L_{b}=$ ' 0 '

$L_{d}=\left\{I_{3}\right\}+\left\{I_{2}\right\}+L_{2}$, if $L_{b}={ }^{\prime} 1$ '

Please note that the feedback path will not cause an infinite $L_{d}$ length because of the priority rule or union rule applied to the ' + ' operation.

\section{- Pipeline-flush Pattern}

Name: Pipeline-flush

Description: A control hazard is usually caused by branch instructions. A branch instruction may change the target instruction address to be fetched next. The target address may not be known at the time that the next instruction is fetched. Therefore, the control logic needs to monitor these situations to make sure that the processor works correctly with or without the branch hazards.

Figure 9 shows an example of the branch hazard. The instruction at address 40 compares the register content of $\$ 1$ and $\$ 3$ and jumps to address $72(40+28)$ if $\$ 1=\$ 3$. There are two ways to handle the control hazard: Always Stall and Assume Branch Not Taken.

Always Stall: This is the simplest way to handle the branch hazard. Each time a branch instruction is encountered, the control unit simply stalls the instruction pipeline by injecting a bubble. The control circuit can be implemented similarly to the one shown for data hazard detection. Note that the "Always Stall" strategy does not cause pipeline flush.

Assume Branch Not Taken: Instead of stalling the pipeline immediately, we continue the execution by assuming that the branch will not be taken. If the branch is untaken, the instruction pipeline keeps running without any interruption. If the branch is taken, the instructions that are being fetched and decoded must be discarded. To discard the instructions, we need to change the control code of the instruction in IF, ID, and EX stages (as shown in Figure 10) in such a way that the instruction will not write back any result to the register file or the memory. The control circuit can be implemented as shown in Figure 11. Note that the status register, which decides whether the branch is taken or not, can be set by earlier instruction and is marked by an empty label, or it is set by the branch instruction and is labeled as $L_{l}=\left\{I_{\text {branch }}\right\}$ where $I_{\text {branch }}$ is the branch instruction in MEM stage. 
Program
Execution

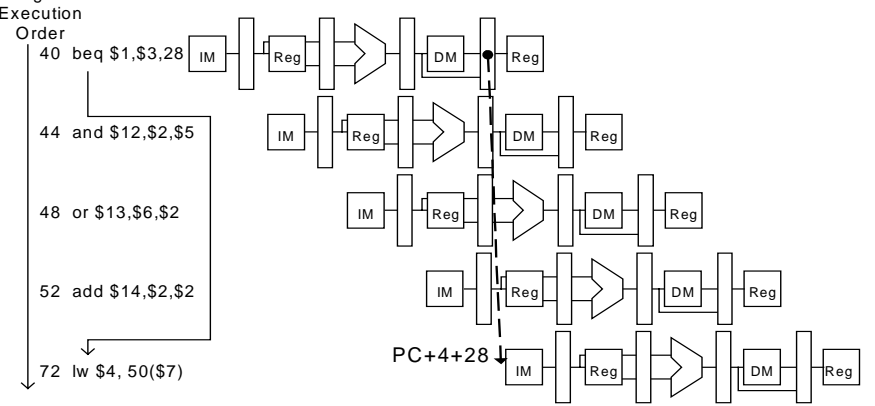

Figure 9 Branch hazard example [11].

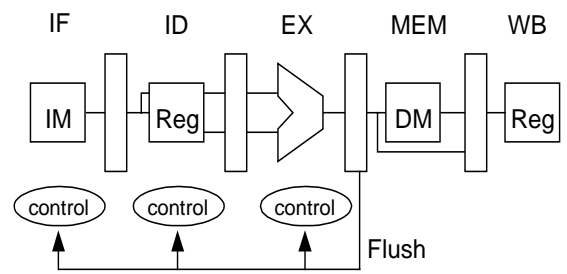

Figure 10 Branch hazard control circuit.

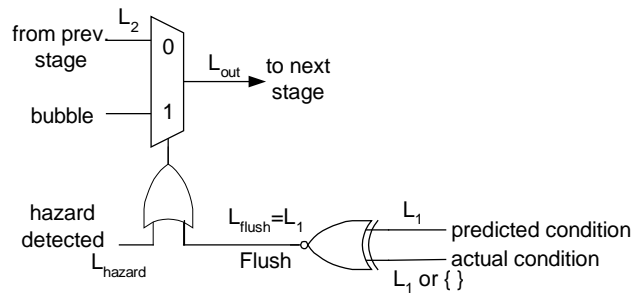

Figure 11 Control circuit for pipeline flush.

Required Rule: (c.f. Figure 11)

- $L_{\text {out }}=L_{1}$ if pipeline is flushed due to the branch misprediction.

- $\quad L_{\text {out }}=L_{2}$ if both "hazard detected" and "Flush" are deasserted.

- $\quad L_{\text {out }}=L_{\text {hazard }}$ if "hazard detected" is asserted.

\section{DESIGN OF THE ZILOG DSP CORE}

The instruction-flow driven power analysis is also useful for power analysis in digital signal processors. Usually the computational resources in a digital signal processor are distributed, and multiple on-chip buses are used to maximize the throughput. Consequently, it is even more difficult to manually perform the labeling. In this section, we use a Zilog voice processor [5] (c.f. Figure 12) as the DSP example for microanalysis. In this processor, there are two on-chip RAM banks: RAM0 and RAM1, a stack, and several distributed registers: X, Y, P and an Accumulator. The lower 64 words of the on-chip RAM can also function as registers. To perform a multiplication, two operands are simultaneously loaded from RAM0 and RAM1 and then stored in X and Y registers within one clock cycle. In the instruction set, $\mathrm{X}$ and $\mathrm{Y}$ can also function as general-purpose registers to move data around. Note that the data outputs of $\mathrm{X}$ and $\mathrm{Y}$ registers are directly tied to the inputs of the multiplier. Therefore, if a 'mov' instruction moves the data from Accumulator to $\mathrm{X}$ without the need to perform a multiplication, then the multiplier will still dissipate (waste) power because its inputs change. Our labeling scheme could simply propagate nonmultiple instruction to $\mathrm{X}$ and $\mathrm{Y}$ and capture the wasted power. A similar problem can be automatically detected for the ALU inputs. For example, if we want to perform the multiplication instruction and the result is written into register $\mathrm{P}$, then the value change in $\mathrm{P}$ may be passed on to the ALU and subsequently cause unnecessary power consumption in the ALU. This can also be detected by label propagation.

Another potential problem is that the select line of the MUX may change value even when no ALU instruction is being executed. This problem may be caused by, for example, poorly designed decoder logic. By labeling, we can easily identify the specific part of the instruction decoder that causes this problem. This last case also shows that the instruction-labeling scheme can help debug and verify the hardware early in the design process.

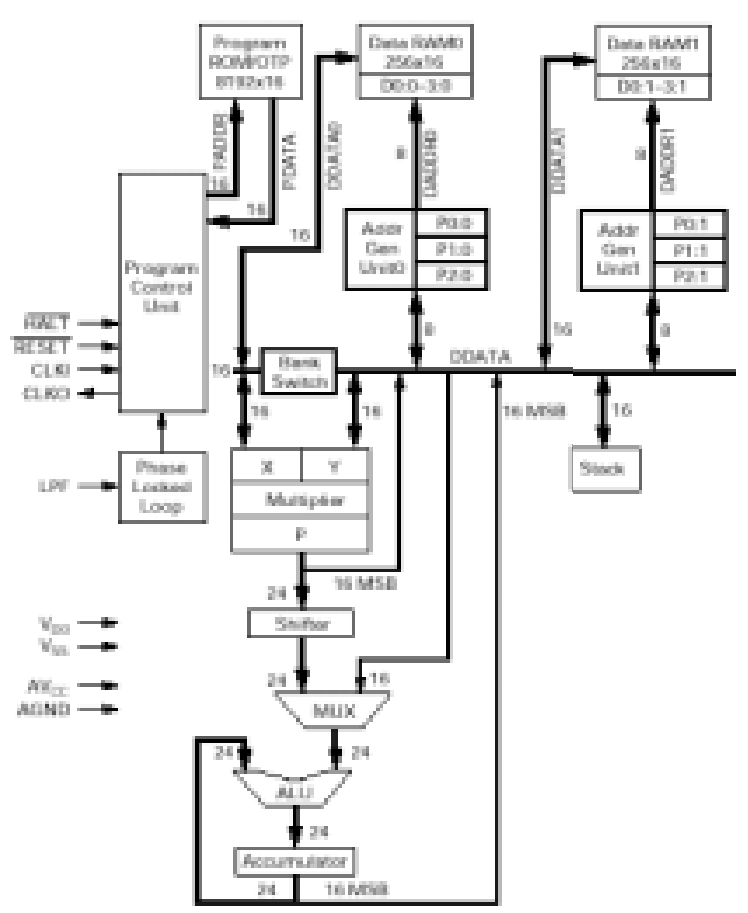

Figure 12 Zilog DSP processor core [5].

We have designed a DSP core, which is compatible with Z89C00 instruction set [6], with Verilog HDL. The Z89C00 DSP instruction set, consisting of 30 basic instructions, is optimized for high code density and reduced execution time. Single-cycle instruction execution is possible on most instructions, including multiplication and I/O operations. There are 9 different addressing modes, which enables high code density.

\section{EXPERIMENTAL SETUP}

\section{- DSP core mapping}

The DSP core is mapped to TSMC Process-Perfect Library [7] with Synopsys Design Compiler v. 1999.05 [8]. The RAM0, RAM1, and instruction memory are not mapped and remain in behavioral model for the purpose of fast RTL simulation. The power consumption inside memory may be captured or estimated separately if a more accurate modeling is needed. We construct the propagation rules with Union Rules for all of the TSMC library cells and verify them with several architectural patterns.

\section{- RTL simulator and label propagation engine}

Verilog simulator [9] is used for RTL simulation. A label propagation engine is built with the Verilog Procedural Interface [10], which provides a mechanism to access the internal simulation data of the Verilog simulator. The engine performs label propagation and generates an instruction power consumption profile (cf. Figure 13). We first simulate one clock cycle and record switching activity of each wire in the mapped net list. We do label propagation at the end of the clock cycle. Note that the 
logic value of each net, which is utilized to perform label propagation, should therefore remain unchanged until the end of the current clock cycle.

The energy consumption is then calculated in the third step. Energy dissipation is dependent on the power-supply voltage, the switching activities, and the internal and output load capacitances. The energy dissipated in each cell in each cycle is calculated by the following equation:

$$
E=E_{\text {Internal }}+E_{\text {External }}
$$

where $E_{\text {Internal }}$ denotes the energy dissipation in the internal capacitances of the cell due to input transitions and $E_{\text {External }}$ denotes the energy dissipation due to transitions at the output of the cell (includes the effects of both input pin capacitances of the fanout gates and the routing capacitance of the net connecting the cell and its fanout gates). Obviously the power dissipation is the product of the energy consumption and the clock frequency.

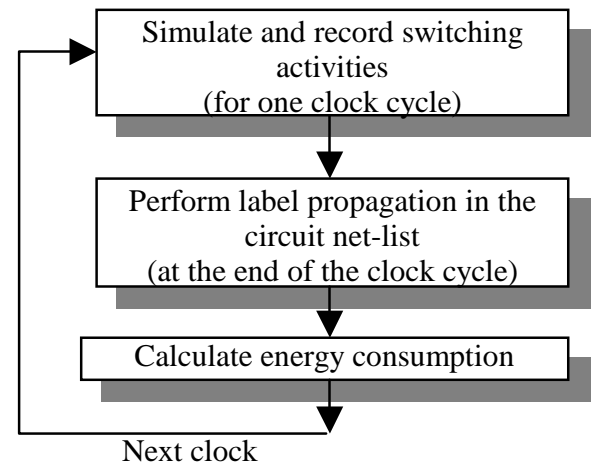

Figure 13 Simulation workflow.

The TSMC data book provides $E_{\text {Internal }}$ and input capacitance values for all cells. For the wire capacitance, we simply assume that it is proportional to the fanout count of the driver. Note that the first part of the equation is the power consumption of the library cell, which is contributed from all instruction labels of its output pins (nets). The second part is the total power consumption of the output nets of the cell in current clock cycle. Iterating the cell instances and summing up their power dissipation $P$, we calculate the total circuit power consumption.

\section{- Target application on Zilog DSP processor}

Currently, we do not have a $\mathrm{C} / \mathrm{C}++$ compiler and assembler for Z89C00 DSP instruction set. Because of the lack of high-level language utilities, it is impossible for us to build complex DSP applications for our testing purpose. Instead, five simple programs were written in assembly language and directly translated into the binary code. This process was cumbersome, but served our goal.

\section{EXPERIMENTAL RESULTS}

The Zilog Z89C00 Instruction Set is categorized into 5 instruction classes, NON, SL, MAC, CTRL, CAS and ALF. NON is for background power consumption, which cannot be attributed to any instruction class. SL is for load and store instructions including different addressing modes. MAC is for simultaneous multiplication and addition instructions. CTRL is for control related instructions. CAS is for comparison and integer arithmetic instructions. ALF is for logical operation instructions.

Five simple programs are used as target applications on the Zilog DSP core. The energy consumption of instruction classes for each program is shown in Figure 14 to Figure 18. Notice that the energy consumption does not include the dissipation in the instruction memory and data memory. The average energy for 'NON' class is the total background energy divided by the total instruction number.

\begin{tabular}{|c|c|c|}
\hline $\begin{array}{c}\text { Instruction } \\
\text { Class }\end{array}$ & $\begin{array}{c}\text { Average } \\
\text { Energy }\left(\mathbf{1 0}^{-8} \mathbf{J}\right)\end{array}$ & $\begin{array}{c}\text { Instruction } \\
\text { Count }\end{array}$ \\
\hline NON & 0.0053 & - \\
\hline SL & 0.0262 & 83 \\
\hline MAC & 0.0513 & 132 \\
\hline CTRL & 0.0101 & 30 \\
\hline CAS & 0.0147 & 7 \\
\hline ALF & 0.0198 & 14 \\
\hline
\end{tabular}

Figure 14 Instruction class energy for program 1.

\begin{tabular}{|c|c|c|}
\hline $\begin{array}{c}\text { Instruction } \\
\text { Class }\end{array}$ & $\begin{array}{c}\text { Average } \\
\text { Energy }\left(\mathbf{1 0}^{-\mathbf{8}} \mathbf{J}\right)\end{array}$ & $\begin{array}{c}\text { Instruction } \\
\text { Count }\end{array}$ \\
\hline NON & 0.0046 & - \\
\hline SL & 0.0128 & 70 \\
\hline MAC & 0.0649 & 35 \\
\hline CTRL & 0.0155 & 125 \\
\hline CAS & 0.0111 & 8 \\
\hline ALF & 0.0124 & 27 \\
\hline
\end{tabular}

Figure 15 Instruction class energy for program 2.

\begin{tabular}{|c|c|c|}
\hline $\begin{array}{c}\text { Instruction } \\
\text { Class }\end{array}$ & $\begin{array}{c}\text { Average } \\
\text { Energy }\left(\mathbf{1 0}^{-\mathbf{8}} \mathbf{J}\right)\end{array}$ & $\begin{array}{c}\text { Instruction } \\
\text { Count }\end{array}$ \\
\hline NON & 0.0071 & - \\
\hline SL & 0.0193 & 20 \\
\hline MAC & 0.0674 & 98 \\
\hline CTRL & 0.0138 & 58 \\
\hline CAS & 0.0265 & 142 \\
\hline ALF & 0.0136 & 33 \\
\hline
\end{tabular}

Figure 16 Instruction class energy for program 3.

\begin{tabular}{|c|c|c|}
\hline $\begin{array}{c}\text { Instruction } \\
\text { Class }\end{array}$ & $\begin{array}{c}\text { Average } \\
\text { Energy }\left(\mathbf{1 0}^{-\mathbf{8}} \mathbf{J}\right)\end{array}$ & $\begin{array}{c}\text { Instruction } \\
\text { Count }\end{array}$ \\
\hline NON & 0.0054 & - \\
\hline SL & 0.0210 & 61 \\
\hline MAC & 0.0477 & 10 \\
\hline CTRL & 0.0144 & 78 \\
\hline CAS & 0.0120 & 17 \\
\hline ALF & 0.0188 & 104 \\
\hline
\end{tabular}

Figure 17 Instruction class energy for program 4.

\begin{tabular}{|c|c|c|}
\hline $\begin{array}{c}\text { Instruction } \\
\text { Class }\end{array}$ & $\begin{array}{c}\text { Average } \\
\text { Energy(10 } \\
\left(\mathbf{1 0}^{-8} \mathbf{J}\right)\end{array}$ & $\begin{array}{c}\text { Instruction } \\
\text { Count }\end{array}$ \\
\hline NON & 0.0058 & - \\
\hline SL & 0.0190 & 56 \\
\hline MAC & 0.0520 & 42 \\
\hline CTRL & 0.0171 & 70 \\
\hline CAS & 0.0201 & 33 \\
\hline ALF & 0.0179 & 42 \\
\hline
\end{tabular}

Figure 18 Instruction class energy for program 5.

\section{CONCLUSIONS}

An instruction-flow-based power analysis technique was proposed to accurately calculate power dissipation induced by a certain instruction running on a target processor. The proposed algorithm automatically attributes the power consumption of each gate within the processor to the instructions that are being executed in the instruction pipeline or instruction execution pool. As a result, the power microanalysis enables the processor architect or designer to identify the instructions in the instruction set that 
consume a lot of power or, more importantly, waste power. When some component does not work as expected from a power, performance, or functionality perspective, the instruction-labeling scheme can help designers trace the problem back to the instructions that caused the problem. The proposed technique also helps in synthesizing an instruction-level power macromodel, which can later be used by a compiler to generate power-efficient executables.

\section{REFERENCES}

[1] C-L. Su, C-Y. Tsui and A.M. Despain, "Low Power Architecture Design and Compilation Techniques for High Performance Processors," Proc. of IEEE COMPCON, 1994, pages 489-498.

[2] V. Tiwari, S. Malik, A. Wolfe, and T.C. Lee, "Instruction Level Power Analysis and Optimization of Software," J. of VLSI Signal Processing, Vol. 13, No. 2/3, August 1996, pages 223-238.

[3] E. Macii, M. Pedram and F. Somenzi, "High level power modeling, estimation and optimization," IEEE Trans. on Computer Aided Design, Vol. 17. No. 11, November 1998, pages 1061-1079.

[4] K. Roy and S. C. Prasad, Low Power CMOS VLSI Circuit Design, John Wiley and Sons, 2000.

[5] "Z89223/273/323/373 16-Bit Digital Signal Processors with A/D Converter: Production Specification," URL: http://www.zilog.com.

[6] "Zilog Z89C00 Instruction Set,” URL: http://www.zilog.com.

[7] TSMC Process-Perfect Library Data Book 2.5-Volt Standard Cell TSMC $0.25 \mu \mathrm{m}$ Process, January 1999.

[8] Synopsys Design Compiler Reference Manual, v. 1999.05, Synopsys, 1999.

[9] Verilog-XL Reference Manual, Cadence, 1998.

[10] VPI User Guide and Reference, Cadence, 1998.

[11] D. A. Patterson, J. L. Hennessy, "Computer Organization \& Design, The Hardware/Software Interface," Morgan Kaufmann, 1994. 